\title{
Q148N, a Novel Integrase Inhibitor Resistance Mutation Associated with Low-Level Reduction in Elvitegravir Susceptibility
}

\author{
Vici Varghese, Benjamin A. Pinsky,,2 Darvin S. Smith,, Daniel Klein, and Robert W. Shafer ${ }^{1}$
}

\begin{abstract}
The integrase strand transfer inhibitor (INSTI)-resistance mutations Q148H/K/R are arguably the most important INSTI-resistance mutations as they represented the first step to high-level dolutegravir cross-resistance. We describe an individual with transmitted four-class drug resistance whose virus sequence had the previously uncharacterized mutation Q148N. Infectious molecular HIV-1 clones containing Q148N alone and in combination with G140S demonstrated $\sim 2.4-4.5$ reduced elvitegravir susceptibility depending on the virus's genetic context but retained susceptibility to raltegravir and dolutegravir. This level of reduced elvitegravir susceptibility is lower than that observed with $\mathrm{Q} 148 \mathrm{H} / \mathrm{K} / \mathrm{R}$ and in fact the infected individual responded to an initial treatment regimen containing tenofovir/emtricitabine/elvitegravir/cobicistat. Q148N was associated with a higher replication capacity than Q148H, suggesting that this mutation may be more fit in the absence of selective INSTI therapy.
\end{abstract}

A 50-YEAR-OLD HisPaniC homosexual man underwent HIV-1 seroconversion in August 2014. His CD4 count at the time of diagnosis was 405 cells $/ \mathrm{mm}^{3}$ and his plasma HIV-1 RNA level was 3,980 copies/ml. A baseline genotypic resistance test revealed the nucleoside reverse transcriptase inhibitor-resistance mutations L210W and T215D that were interpreted as causing intermediate resistance to zidovudine and low-level resistance to tenofovir; the non-nucleoside reverse transcriptase inhibitor (NNRTI)-resistance mutations $\mathrm{K} 101 \mathrm{P}$ and K103S that were interpreted as causing high-level resistance to each of the NNRTIs; and the protease inhibitorresistance mutations D30N, L33F, I54V, N88D, and L90M that were interpreted as causing intermediate or high-level resistance to each of the PIs except darunavir. The patient was started on the once daily integrase strand transfer inhibitor (INSTI)-containing fixed-dose combination tenofovir/emtricitabine/elvitegravir/cobicistat.

In November 2014, the patient changed clinics and an INSTI resistance test was ordered on his August 2014 sample. The test revealed G140S, a nonpolymorphic accessory INSTI-resistance mutation, and Q148N, a previously uncharacterized mutation in the INSTI-binding pocket of integrase enzyme. At the same time, the plasma HIV-1 RNA level was $<50$ copies $/ \mathrm{ml}$ and CD4 count was 767 cells $/ \mathrm{mm}^{3}$. Although the patient's genotypic resistance test was reported as being associated with intermediate resistance to raltegravir and elvitegravir based on the presence of G140S, no change to therapy was made because of the patient's virological and immunological response to therapy. The patient has since been monitored closely and multiple subsequent plasma HIV-1 RNA levels have been $<50$ copies/ml.

In 2015, we created a panel of infectious molecular clones to assess the effect of the novel mutation Q148N on INSTI susceptibility. The panel included site-directed HIV-1 mutants containing G140S+Q148N, Q148N alone, G140S+ $\mathrm{Q} 148 \mathrm{H}$, and Q148H alone. It also contained a recombinant infectious molecular clone from the patient's August 2014 sample and clones in which G140S alone and G140S and Q148N were independently mutated back to the wild type residues at these positions. These clones were subjected for phenotypic susceptibility testing using the PhenoSense assay (Monogram). ${ }^{1}$

Q148N \pm G140S was associated with 2.4- to 4.5-fold reduced susceptibility to elvitegravir but not to raltegravir or dolutegravir (Table 1). This level of INSTI resistance was lower than that observed with Q148H alone (19-fold reduced

\footnotetext{
${ }^{1}$ Division of Infectious Diseases, Department of Medicine, Stanford University, Stanford, California.

Departments of ${ }^{2}$ Pathology and ${ }^{3}$ Microbiology and Immunology, Stanford University, Stanford, California.

${ }^{4}$ Kaiser Permanente Medical Care Program-Northern California, Oakland, California.
}

(C) Vici Varghese et al., 2016; Published by Mary Ann Liebert, Inc. This Open Access article is distributed under the terms of the Creative Commons Attribution Noncommercial License (http://creativecommons.org/licenses/by-nc/4.0/) which permits any noncommercial use, distribution, and reproduction in any medium, provided the original author(s) and the source are credited. 
Table 1. Integrase Strand Transfer Inhibitor Resistance Mutations And IN Vitro Susceptibility Results Of Virus Clones With AND Without Q148N

\begin{tabular}{llcccc}
\hline $\begin{array}{l}\text { IN resistance } \\
\text { mutations }\end{array}$ & Background $^{\mathrm{a}}$ & $\begin{array}{c}\text { RAL (fold } \\
\text { change })\end{array}$ & $\begin{array}{c}\text { EVG (fold } \\
\text { change })\end{array}$ & $\begin{array}{c}\text { DTG (fold } \\
\text { change })\end{array}$ & RC $(\%)$ \\
\hline None & NL43 & 0.9 & 0.8 & 0.9 & NA \\
Q148N & NL43 & 0.7 & 2.4 & 0.6 & 88 \\
G140S, Q148N & NL43 & 0.6 & 3.1 & 0.6 & NA \\
Q148H & NL43 & 19 & 5.5 & 0.6 & 27 \\
G140S, Q148H & NL43 & $>150$ & $>150$ & 3.4 & NA \\
G140S, Q148N & Clinical sample & 0.6 & 4.2 & 0.5 & NA \\
Q148N & Clinical sample & 1.0 & 4.5 & 0.7 & NA \\
None & Clinical sample & 1.0 & 1.3 & 1.1 & \\
\hline
\end{tabular}

${ }^{\text {a }}$ Site-directed mutants were created using the HIV-1 plasmid pNL43 and using a recombinant infectious clone created from the PCRamplified cDNA extracted from plasma virus. All mutated clones were transfected and sequenced before susceptibility testing using previously published methods. ${ }^{14}$ Susceptibility testing and RC determination were determined using the PhenoSense assay (Monogram). ${ }^{1}$

$\mathrm{RC}$, replication capacity.

raltegravir susceptibility and 5.5-fold reduced elvitegravir susceptibility) or Q148H+G140S (>150-fold reduced susceptibility to raltegravir and elvitegravir and 3.4-fold reduced dolutegravir susceptibility). Among the site-directed mutants, Q148N had a higher replication capacity than Q148H ( $88 \%$ vs. $27 \%$ ).

\section{Comment}

Q148 is conserved in all lentiviruses and plays an essential role in integrase strand transfer activity. ${ }^{2,3}$ Mutations at this position are arguably the most important INSTI-associated resistance mutations as they represent the first step in the pathway to high-level dolutegravir cross-resistance. ${ }^{4,5}$ Of 1,120 integrase sequences from patients receiving raltegravir in GenBank as of June 2015, 23\%, 8\%, and 1\% had the previously characterized INSTI-resistance mutations Q148H, Q148R, and Q148K, respectively. ${ }^{6}$

Q148N was present in viruses from three raltegravirexperienced individuals and is the only other mutation at this position detected in GenBank sequences. ${ }^{6,7} \mathrm{Q} 148 \mathrm{~N}$ is also the only mutation other than $\mathrm{Q} 148 \mathrm{H} / \mathrm{R} / \mathrm{K}$ that has been shown to have strand transfer activity in biochemical assays. ${ }^{3}$ There have been too few published sequences obtained from elvitegravir-experienced individuals to determine whether Q148N occurs at a higher frequency after elvitegravir treatment than after raltegravir treatment. The rarity of Q148N and its weaker association with INSTI resistance suggest that it may be a revertant mutation arising from single nucleotide substitutions in viruses that once harbored $\mathrm{Q} 148 \mathrm{H}(\mathrm{CAC}$ or CAT) or Q148K (AAA or AAG) $=>\mathrm{Q} 148 \mathrm{~N}$ (AAC or AAT).

The patient's response to therapy may reflect the relatively low-level reduction in elvitegravir susceptibility associated with Q148N and the relatively low plasma HIV-1 RNA level at the time of diagnosis. However, had a baseline INSTI genotypic resistance test been available and had the effect of Q148N been known, a different regimen would likely have been chosen.

Although transmitted INSTI-associated resistance has been practically absent in surveillance studies, ${ }^{8-10}$ there have been at least three case reports of INSTI-associated TDR before this one ${ }^{11-13}$. This report therefore suggests that baseline INSTI genotypic resistance testing may play an in- creasing role in the future, particularly in patients with transmitted resistance to other drug classes.

\section{Sequence Data}

The sequence of the clinical clone has been submitted to the GenBank under the accession number KU761565.

\section{Acknowledgment}

This study was funded in part by NIAID AI068581.

\section{Author Disclosure Statement}

No competing financial interests exist.

\section{References}

1. Petropoulos CJ, Parkin NT, Limoli KL, et al.: A novel phenotypic drug susceptibility assay for human immunodeficiency virus type 1. Antimicrob Agents Chemother 2000; 44:920-928.

2. Engelman A, Craigie R: Identification of conserved amino acid residues critical for human immunodeficiency virus type 1 integrase function in vitro. J Virol 1992;66:63616369.

3. Johnson AA, Santos W, Pais GC, et al.: Integration requires a specific interaction of the donor DNA terminal $5^{\prime}$ cytosine with glutamine 148 of the HIV-1 integrase flexible loop. J Biol Chem 2006;281:461-467.

4. Seki T, Suyama-Kagitani A, Kawauchi-Miki S, et al.: Effects of raltegravir or elvitegravir resistance signature mutations on the barrier to dolutegravir resistance in vitro. Antimicrob Agents Chemother 2015;59:2596-2606.

5. Eron JJ, Clotet B, Durant J, et al.: Safety and efficacy of dolutegravir in treatment-experienced subjects with raltegravir-resistant HIV type 1 infection: 24-week results of the VIKING Study. J Infect Dis 2013;207:740-748.

6. Rhee SY, Gonzales MJ, Kantor R, Betts BJ, Ravela J, Shafer RW: Human immunodeficiency virus reverse transcriptase and protease sequence database. Nucleic Acids Res 2003;31:298-303.

7. Hurt CB, Sebastian J, Hicks CB, Eron JJ: Resistance to HIV integrase strand transfer inhibitors among clinical specimens in the United States, 2009-2012. Clin Infect Dis 2014;58:423-431. 
8. Casadella M, van Ham PM, Noguera-Julian M, et al.: Primary resistance to integrase strand-transfer inhibitors in Europe. J Antimicrob Chemother 2015;70:2885-2888.

9. Doyle T, Dunn DT, Ceccherini-Silberstein F, et al.: Integrase inhibitor (INI) genotypic resistance in treatmentnaive and raltegravir-experienced patients infected with diverse HIV-1 clades. J Antimicrob Chemother 2015;70: 3080-3086.

10. Stekler JD, McKernan J, Milne R, et al.: Lack of resistance to integrase inhibitors among antiretroviral-naive subjects with primary HIV-1 infection, 2007-2013. Antivir Ther 2015;20:77-80.

11. Boyd SD, Maldarelli F, Sereti I, et al.: Transmitted raltegravir resistance in an HIV-1 CRF_AG-infected patient. Antivir Ther 2011;16:257-261.

12. Volpe JM, Ward DJ, Napolitano L, et al.: Five antiretroviral drug class-resistant $\mathrm{HIV}-1$ in a treatment-naive patient successfully suppressed with optimized antiretroviral drug selection. J Int Assoc Provid AIDS Care 2015;14: 398-401.
13. Young B, Fransen S, Greenberg KS, et al:: Transmission of integrase strand-transfer inhibitor multidrug-resistant HIV1: Case report and response to raltegravir-containing antiretroviral therapy. Antivir Ther 2011;16:253-256.

14. Reuman EC, Bachmann MH, Varghese V, Fessel WJ, Shafer RW: Panel of prototypical raltegravir-resistant infectious molecular clones in a novel integrase-deleted cloning vector. Antimicrob Agents Chemother 2010;54:934-936.

Address correspondence to: Vici Varghese Division of Infectious Diseases Department of Medicine 300 Pasteur Drive, L1C43, Lane Building Stanford University Stanford, CA-94305

E-mail: viciv@stanford.edu 